\title{
Pancreatic Cancer: Pathogenesis, Diagnosis, and Laboratory Tests
}

\author{
Rikarni \\ Department of Clinical Pathology, Faculty of Medicine, Andalas University/Dr. M. Djamil Hospital, Padang, Indonesia. \\ E-mail: rikarni_dr@yahoo.com
}

\begin{abstract}
Pancreatic cancer is the eleventh cause of cancer death in Indonesia in 2020. However, pancreatic cancer is the second leading cause of cancer death in the United States after lung cancer. Although it is substantially less common than the other malignancies, pancreatic carcinoma is near the top of the list of killers because it is a highly aggressive cancer. Pancreatic cancer has multistep carcinogenesis, starting from the Pancreatic Intraductal Neoplasia (PanINs IA, IB, II, and III) and is ended with an invasive neoplastic lesion. The different incidence of pancreatic cancer between countries also shows the important role of environmental factors for the disease. A better understanding of the risk factors, genetics, molecular pathogenesis, symptoms associated with this disease, and the laboratory aspect is essential to inform both health professionals and the general population as the potential preventive and/or early detection measures.
\end{abstract}

Keywords: Pancreatic cancer, multistep carcinogenesis, pathogenesis, diagnosis, CA 19-9

\section{INTRODUCTION}

The pancreas is a transversely oriented retroperitoneal organ extending from the so-called "C loop" of the duodenum to the hilum of the spleen. By blood vessels and adjacent ligaments that serve to line the organs, the pancreas is divided into a head, body, and tail. The pancreas is two organs packaged into one. The islets of Langerhans scattered in the pancreas serve critical endocrine functions, and the exocrine portion that makes up the bulk of this organ is a major source of enzymes that are essential for digestion. ${ }^{1}$

Pancreatic infiltrating ductal adenocarcinoma (more commonly referred to as pancreatic cancer) is the second leading cause of cancer death in the United States, after lung cancer in $2020 .^{2}$ Pancreatic cancer is the eleventh cause of death from cancer in Indonesia, whereas pancreatic cancer is the seventh cause of death from cancer in Asia in 2020.,4 Although it is substantially less common than the other malignancies, pancreatic carcinoma is near the top of the list of killers because it is a highly aggressive carry. ${ }^{1}$

Pancreatic cancer is most frequent in elder people, the risk of developing pancreatic cancer goes up as people age, about $80 \%$ are at least 60 years old and 71 is the average age at the time of diagnosis. It is frequently found at an advanced stage, which contributes to five-year survival rates of $2 \%-9 \%$, ranking firmly the last amount all cancer sites in terms of prognostic outcomes for patients. ${ }^{5,6}$

Pancreatic cancer has multistep carcinogenesis, starting from Pancreatic Intraductal Neoplasia (PanINs IA, IB, II, and III) and is ended with an invasive neoplastic lesion. PanINs progression is supported by a lot of gene mutations. This specific multistep carcinogenesis suggests that diagnosis at early stage lesion by PanINs detection will likely stop the progression. This is the safer way to change the natural history of pancreatic cancer. Unfortunately, early-stage pancreatic cancer commonly does not show clinical manifestation, suggesting the need for an improved method of early detection of precursors. ${ }^{6}$

\section{DEFINITION}

Pancreatic cancer usually refers to ductal adenocarcinomas of the pancreas because more than $90 \%$ of pancreatic tumors arise from the ductal epithelium. ' About its characteristics, pancreatic cancer has high invasiveness and the tendency to infiltrate peri-pancreatic tissues. In addition, it has a high trend to show nodal metastasis (peri-pancreatic, gastric, mesenteric, omental, and peri-portal nodes) and hepatic, bone, or pulmonary metastasis. $^{6}$ 


\section{Epidemiology}

The epidemiological data in Italy in 2017 shows more than 13,000 new cases and more than 11,000 deaths in 2015;22/100,000 new cases per year and in 2016 it increased in both gender, but a higher number was found in the male population. ${ }^{6}$ The incidence in the United States was 56,654 new cases and 47,683 death in $2020{ }^{2}$ The incidence was 6,485 new cases and 16,167 death in South-Eastern Asia. ${ }^{8}$ The number of new cases of pancreatic cancer in Indonesia in 2020 was 5,781 cases, the incidence is $1.5 \%$ among cancers and 5,690 deaths, making it the top eleventh cause of death from cancer. ${ }^{3}$

The low survival rate for pancreatic cancer has been shown by data in a study by Globocan in 2018, which showed that the number of new cases $(458,918$ cases) of pancreatic cancer from 185 countries was similar to the number of deaths (432,242 deaths). The main cause contributing to a low survival rate is the diagnosis of pancreatic cancer in its advanced stages (52\% for advanced stages and $9 \%$ for the initial stages) for whom the 5 -year-survival rate is only $2-5 \%$. The mortality of cancer is predominantly caused by multi-organ failure due to metastasis. ${ }^{9}$

\section{Risk Factor}

The different incidence of pancreatic cancer between countries also shows the important role of environmental factors for the disease. The risk factors are divided into non-modifiable and modifiable risk factors. ${ }^{5}$

Non-modifiable risk factors are as follows: Age: Most patients are diagnosed at $>50$ years of age, with peak incidence in the seventh and eighth decades of life. ${ }^{10}$ Gender: The incidence of cancer in males is higher than in females (ASR 5.5 in males and 4.0 in females; Ethnicity: $50-90 \%$ increased risk of pancreatic cancer is found African-Americans compared to Caucasians in the United States; Blood group: Patients with blood group A or B are at a significantly higher risk of developing pancreatic than patients with blood group O; Family history and genetic susceptibility: Patients with familial risk factors have a nine times higher risk of developing pancreatic cancer than those with no family history; Diabetes: Diabetes is a well-established risk factor for pancreatic cancer. Pancreatic cancer can manifest as new onset of diabetes. It is predicted that there will be a higher interest in $\mathrm{HbAlc}$ as a new potential biomarker for pancreatic cancer in the future; Gut Microbiota: A systematic review showed that lower levels of Neisseria elongate and Streptococcus mitis, and higher levels of Porrphyromonas ginggivalis and Granulicatella adiacens are associated with an increased risk of pancreatic cancer. ${ }^{5}$

Modifiable risk factors are as follows: Smoking: Smokers have two to three times higher risk of developing pancreatic cancer compared to non-smokers ${ }^{10}$; Alcohol: Excessive alcohol consumption is also a major cause of chronic pancreatitis, a known risk factor for pancreatic cancer. Therefore, alcohol in this setting is a risk factor for pancreatic cancer; Chronic pancreatitis: If appropriate screening tests can be found, chronic pancreatitis patients can be a potential group for pancreatic cancer screening; Obesity: The World Cancer Research Fund reports an association between pancreatic cancer and increased Body Mass Index (BMI). Given the strength of the evidence linking obesity to pancreatic cancer, it is likely that the rising incidence of obesity is the main cause of the increased incidence of pancreatic cancer in the developed world; Dietary factors: People who consume red and processed meat in excessive amounts have the potential to form carcinogens such as $\mathrm{N}$-nitroso compounds and DNA damage; Infection: Increased risks pancreatic cancer observed in patients with Helicobacter pylori or hepatitis C infections. $^{5}$

\section{Genetics Pancreatic Cancer}

Pancreatic cancer is caused by inherited (germline) and acquired (somatic) mutations in cancer-causing genes. Oncogenes and tumor suppressor genes have been demonstrated to be involved in the development of pancreatic cancer, both by contributing to the growth of the tumor itself as well as to the surrounding microenvironment. ${ }^{7}$

The recent sequencing of the pancreatic cancer genome has confirmed that four genes are most commonly affected by somatic mutation this neoplasm: Kirsten Rat Sarcoma oncogene homolog (KRAS), Cyclin-Dependent Kinase Inhibitor 2A (CDKN2A/p16), Mothers Against Decapentaplegic Homolog 4 (SMAD4), and Tumor protein 53 (TP53). In addition, a few at around 10\% prevalence (e.g., KDM6A, RBM10, MLL3), ${ }^{8,11}$ KRAS is the most frequently altered oncogene in pancreatic cancer, it is activated by a point mutation in greater than $90 \%$ of cases. ${ }^{8}$ These mutations impair the intrinsic GTPase activity of the KRAS protein. In turn, KARS activates some intracellular signaling pathways that promote carcinogenesis. ${ }^{1}$

The $16 / C D K N 2 A$ gene is the most frequently inactivated tumor suppressor gene in pancreatic 
cancer, being inactive in $95 \%$ of cases. The p16 protein has a critical role in cell-cycle control, and its inactivation removes an important checkpoint. The SMAD4 tumor suppressor gene is inactivated in 55\% of pancreatic cancers but rarely found in other tumors, it codes for a protein that plays an important role in downstream signal transduction of the transforming growth factor- $\beta$ receptor. Inactivation of the TP53 tumor suppressor gene occurs in $50 \%$ to $70 \%$ of pancreatic cancers. Its gene product, p53, acts both to enforce cell cycle checkpoints and as an inducer of apoptosis or senescence. Breast Cancer Type 2 susceptibility protein (BRCA2) is also mutated late in a subset of pancreatic cancer. ${ }^{1}$

Global genomic sequencing of human pancreatic adenocarcinoma revealed the extreme complexity of tumor genetics but did identify a core set of only 12 cellular signaling pathways and processes that were each altered in up to $100 \%$ of the tumors. Five signaling pathways affect specific cellular functions, including apoptosis, DNA damage repair, G1/S phase cell cycle progression, cell-cell adhesion, and invasion (Table 1). ${ }^{12}$

\section{Pathogenesis of pancreatic cancer}

There is a progressive accumulation of genetic changes in the pancreatic epithelium as it proceeds from non-neoplastic to noninvasive precursor lesions, to invasive carcinoma. The development and progression of pancreatic cancer is a multistep process. Precursor lesions for pancreatic adenocarcinoma include Intraepithelial Pancreatic Mucinous Neoplasms (IPMNs) and Pancreatic Intraepithelial Neoplasia (PanIN). The most common antecedent lesion of pancreatic cancer arises in small ducts and ductules and is called PanIN ${ }^{1,13}$ Pancreatic cancer progresses from PanIN lesions to invasive carcinoma (Figure 1). Based on cellular and nuclear atypical grades, PanIN lesions are divided into low-grade (PanIN-1A/B) to high (PanIN-3) lesions. ${ }^{14,15}$

Several changes, which occur in key genes (KRAS, CDKN2, TP53, SMAD4/DPC4,BRCA2) are accumulated. Deregulated signaling pathways, stromal associated factors, and mRNAs serve as fuel for the development pancreatic cancer. KRAS is mutated, oncogenic miRNAs are overexpressed, and stromal associated factors are activated in PanIN-1 lesions. Mucin 1 is overexpressed, and inactivating mutations in the p16/CDKN2A gene are observed in PanIN-2 lesions. Finally, PanIN-3 lesions are associated with inactivating mutations in TP53, BRCA2, and SMAD4. ${ }^{12,15}$

\section{Role of Tumor microenvironment in pancreatic cancer pathogenesis}

Pancreatic tumors are not only composed of tumor cells but are marked by several cell populations, such as fibroblasts, immune cells, and endothelial cells. Growing evidence suggests that a major role in tumorigenesis is tumor-stromal interactions, both at primary and secondary sites. CXCL12 is taken up by tumor cells and promotes tumor progression, metastasis, and chemoresistance. Pancreatic tumor cells produce Sonic hedgehog (Shh). Overexpression of Shh is detectable as early as PanIN1 and through all disease progression.

In the cancer cell, Shh expression is induced by KRAS and NFkB pathways. As a results, Shh can either activate $\mathrm{Hh}$ signalling in the cancer cell or the stromal cell, through canonical $\mathrm{Hh}$ signalling. Other signalling pathways, such as KRAS, can also induce

Table 1. Commonly affected signaling pathways and the most commonly affected genes from these pathways in pancreatic cancer ${ }^{12}$

\begin{tabular}{ll}
\hline Signaling Pathway & Affected Gene(s) (Chromosomal Region) \\
\hline Apoptosis & TP53 (17p) \\
DNA damage repair & TP53 (17p) \\
G1/S phase transition & CDKN2A/p16 (9p) \\
& CCND1 (11q22) \\
Cell-cell adhesion & \\
Regulation of invasion & CDH1 (16q22) \\
Integrin signaling & \\
Homophilic cell adhesio & \\
Embryonic signaling & \\
Notch pathway & \\
Hedgehog pathway & \\
Wnt pathway & \\
MAPK signaling & \\
c-Jun N-terminal kinase & \\
ERK & K-ras2 (12p) \\
TGF- $\beta$ signaling & SMAD4/DPC4 (18q) \\
\hline
\end{tabular}




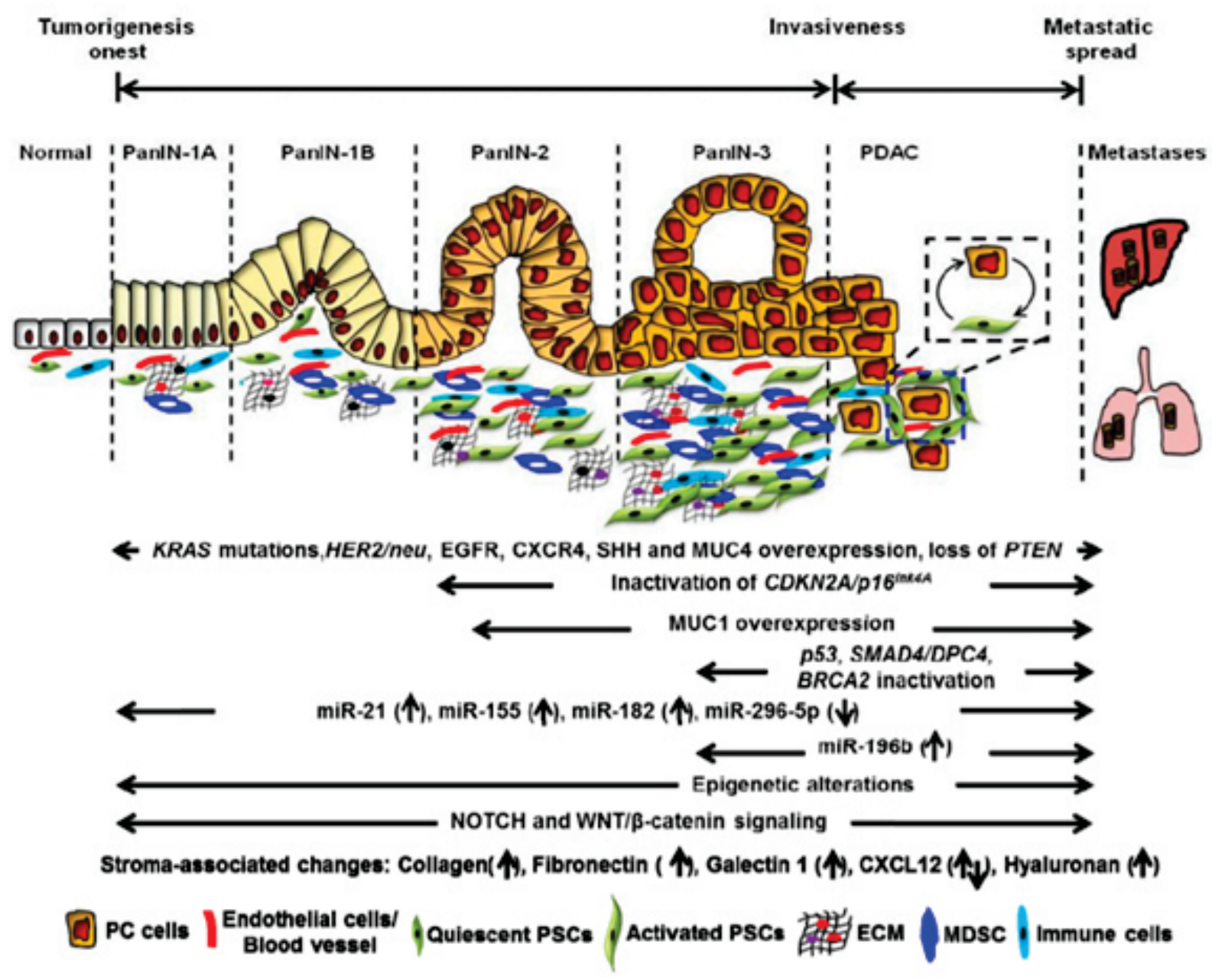

Figure 1. Histopathological and molecular changes in the pathogenesis of pancreatic cancer. Kirsten rat sarcoma oncogene homolog (KRAS), Cyclin-dependent kinase inhibitor 2 (CDKN2), Tumor protein 53 (TP53), Mother against decapentaplegic homolog 4/Deleted in pancreatic cancer 4 (SMAD4/DPC4), Breast cancer type 2 (BRCA2), Human epidermal growth factor receptor 2 (Her-2/neu), Epidermal growth factor receptor (EFGR), chemokine receptor type 4 (CXCR4), Sonic hedgehog (Shh), Mucin 4 (MUC4), Phosphatase and tensin homolog (PTEN), Motif chemokine $12\left(\right.$ CXCL12) ${ }^{15}$

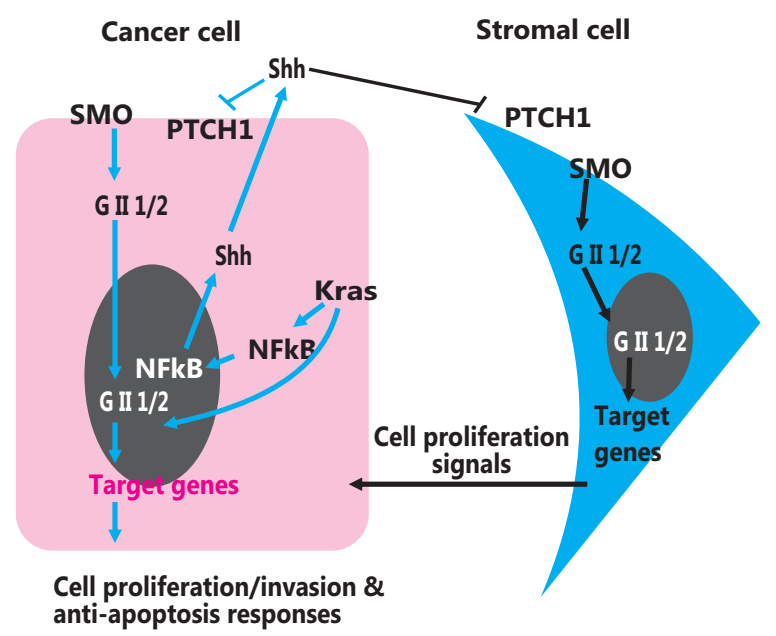

Figure 2. Hh signalling in the cancer and stromal cells ${ }^{14}$
Gli transcriptional activity. As a results, cancer cell will be more proliferative, more invasive, and more resistant to apoptosis. Hh signalling activation in the stromal cells can feedback to stimulate cancer cells proliferation (Figure 2). ${ }^{14}$

\section{Metabolism in Pancreatic Cancer}

The variability of desmoplasia triggered by stromal cells further complicates the reconstruction of a metabolic view of this cancer. Metabolic clinical positron emission tomography scanning shows that the cancer is metabolically active when corrected for variable low perfusion. Recent studies suggest that pancreatic cancer metabolism should be viewed as a composite picture based on the pancreatic ductal adenocarcinoma cells themselves rather than a unidimensional phenotype. Furthermore, additional 


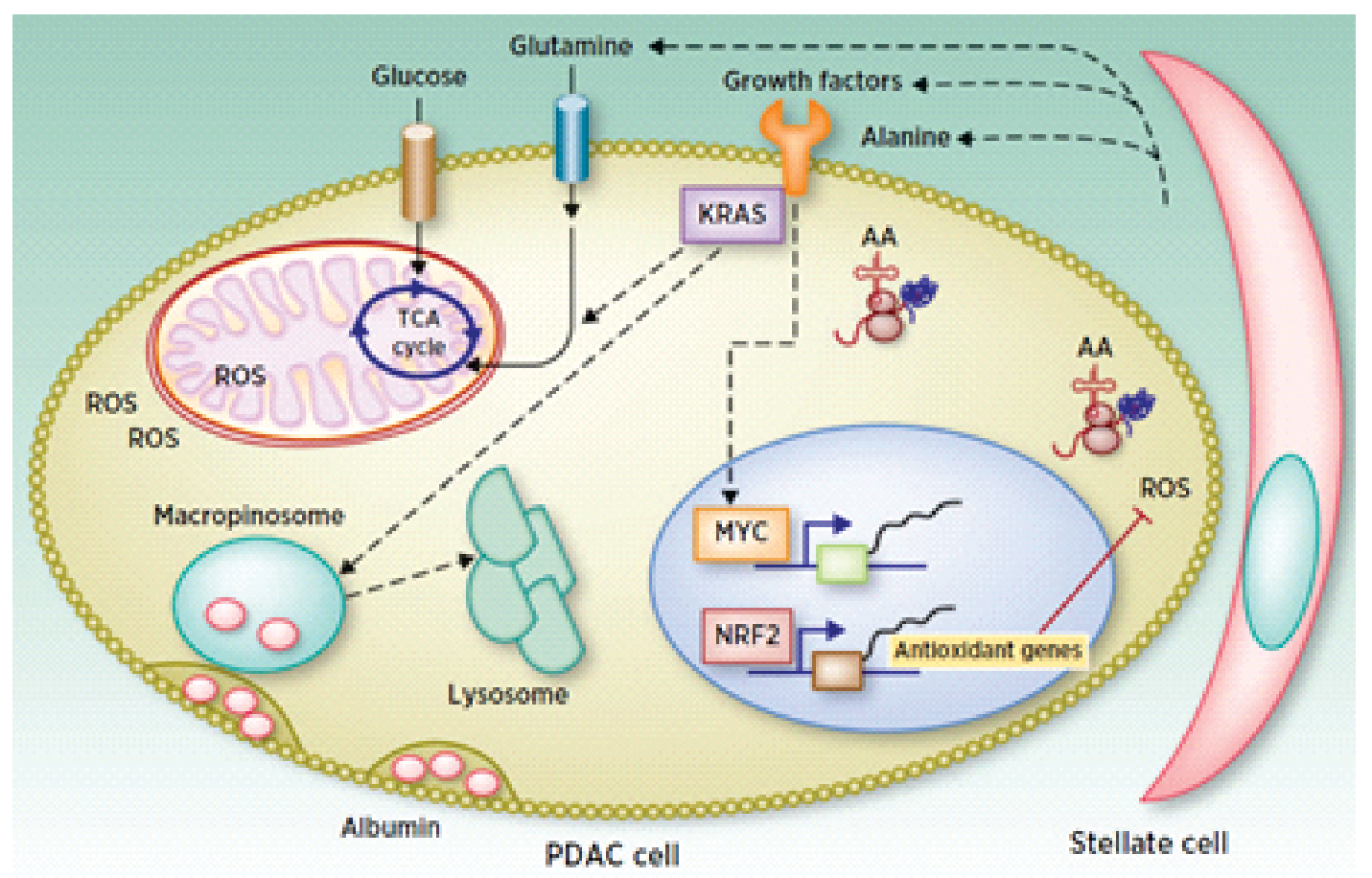

Figure 3. Pancreatic ductal adenocarcinoma metabolism. The interplay between adenocarcinoma cells and stellate cells. Tricarboxylic acid (TCA) cycle. Pancreatic adenocarcinoma (PDAC) ${ }^{16}$

metabolic susceptibility may exist for therapeutic interventions beyond those of the pancreatic cancer cell (Figure 3). ${ }^{16}$

In pancreatic ductal adenocarcinoma cells, metabolic changes occur by activation of KRAS and MYC, which promote glutaminolysis, glycolysis, and macropinocytosis (RAS-mediated phenotype that increases the consumption of proteins such as albumin, which will be digested by lysosomal enzymes to promote pancreatic cancer cell growth). NRF2 as a key transcription factor with oxidative stress from this metabolism modulates redox homeostasis for pancreatic cancer cell survival. The stellate cell is depicted to modulate pancreatic cancer survival through the presence of growth factors and nutrients (Figure 3). ${ }^{16}$

\section{Signs and Symptoms}

Pancreatic cancer rarely manifests specific signs or symptoms. Because of its atypical symptoms, it is frequently diagnosed in an advanced stage. ${ }^{6}$ The early clinical manifestations of pancreatic cancer are often vague, such as nonspecific gastrointestinal complaints (nausea, unexplained abdominal pain), fatigue, and increased weight loss. ${ }^{6,7}$ Diagnostic tests are often epigastric pain and obstructive ileus but are often late symptoms associated with advanced local or regional disease. Because approximately $75 \%$ of pancreatic carcinomas are located in the head of the pancreas, clinical presentations are often related to compression or invasion of the biliary tree or pancreatic ducts. ${ }^{7,17}$ New-onset diabetes mellitus could be found with pancreatic cancer in one fourth of patients, particularly in patients over 50 years old, and glucose intolerance can be observed in two-thirds of patients. ${ }^{12}$

Courvoisier's sign can be found in less than a third of patients. Patients whose also pancreatic duct obstruction will exhibit pancreatic exocrine insufficiencies such as steatorrhea and malabsorption. Extrapancreatic extension in distal tumors causes invasion of the spleen, stomach, splenic flexure of the colon, or left adrenal gland. ${ }^{12}$

\section{DIAGNOSIS}

Transabdominal Ultrasonography is often used for patients with pancreatic cancer with jaundice. Ultrasonography can evaluate the presence of gallstones and confirm biliary dilation. CT is the method of choice for cancer stage and diagnosis. The latest upgrade in this technology has greatly improved the resolution with faster and thinner part imaging. Multiphase imaging technique use 
precontrast, early phase describes the tumor, while the late phase increases vascular connections and liver metastases. Sensitivity of CT ranges from $86 \%$ to $97 \%$, but may reach $77 \%$ for lesions less than $2 \mathrm{~cm} 77 \%{ }^{12}$

Compared to Computed Tomography (CT) and MRI, Endoscopic Ultrasound (EUS) has a higher sensitivity for identifying solid pancreatic lesions less than $2 \mathrm{~cm}$. International Cancer of the Pancreas Screening Consortium group recommends a combination of EUS and Magnetic Resonant Imaging (MRI)/Magnetic Resonance Cholangiopancreatography (MRCP) for screening purposes and to avoid the risk of ionizing radiation. ${ }^{5}$ Endoscopic ultrasound may provide additional information, which is useful for preoperative assessment, including fine-needle aspiration biopsy under endoscopic guidance. ${ }^{7}$ CT scans can provide information regarding the presence of metastatic disease, vascular invasion, and potential for resection. ${ }^{7.12}$ Positron Emission Tomography (PET) provides metabolic rather than tumor morphology information. PET is a non-invasive imaging instrument, and its method is based on the greater utilization of glucose by tumor cells than normal pancreatic parenchyma. ${ }^{12}$

\section{LABORATORY TESTS OF PANCREATIC CANCER}

\section{Tumor Marker}

\section{a. Carbohydrate antigen (CA 19-9)}

The most clinically useful serum tumor marker for pancreatic cancer is Carbohydrate antigen 19-9 (CA 19-9), a sialylated Lewis antigen (Lea) commonly produced in pancreatic and hepatobiliary disease but also increased in many other conditions. The only marker approved by the United States Food and Drug Administration for use in the routine management of pancreatic cancer is serum cancer antigen 19-9.5 In addition, Ca 19-9 levels may be elevated in colorectal, gastric, hepatocellular, esophageal, and ovarian cancer. A non-malignant condition that may cause increases of $\mathrm{Ca}$ 19-9 are acute cholangitis, cholestasis, jaundice, and pancreatitis. ${ }^{17}$ The low positive predictive value of CA 19-9 indicates that it cannot be used in screening, but it can contribute to making a diagnosis in symptomatic patients. ${ }^{5,18}$ Unfortunately, CA19-9 lacks sensitivity or specificity for early pancreatic cancer diagnosis, despite its routine use to monitor disease progression, recurrence, and/or therapy response..$^{10,14}$
Pre-operative CA 19-9 levels correlate with staging and severity of the disease, indicating that they can complement information from imaging, laparoscopy, and biopsy to determine the resectability of a pancreatic tumor. In patients without biliary obstruction, a high CA 19-9 levels can be used as an indication for staging laparoscopy. Post-operative CA 19-9 levels may also help to predict the benefit of adjuvant therapy. ${ }^{18}$

\section{b. Carcinoembryonic antigen (CEA)}

The plasma concentration of the tumor markers Carcinoembryonic Antigen (CEA) may be elevated in up to $50 \%$ of patients with pancreatic malignancy, but they can be elevated with other (particularly colorectal) cancers and sometimes in non-malignant disease. Unfortunately, pancreatic cancer often presents late; by the time it is diagnosed, metastases are often present. ${ }^{17}$ In addition, CEA levels may be elevated in colorectal, breast, gastric, lung, mesothelioma, esophageal gastric, hepatocellular, esophageal, and ovarian cancer. A non-malignant condition that may increase CEA levels are chronic liver disease, colitis, diverticulitis, irritable bowel syndrome, and jaundice. ${ }^{18}$

\section{c. Other tumor markers}

The presence of circulating tumor cells originating from pancreatic cancer can be diagnostic, but it is present only in a few patients with metastatic disease. In contrast, circulating tumor DNA encoding mutant KRAS has been detected at the time of diagnosis in $43 \%$ of patients with localized disease, suggesting that circulating tumor DNA consisting of a panel of mutated genes such as mutated KRAS or mutated TP53 may serve as a non-invasive early diagnostic test. ${ }^{10}$ The presence of DNA mutations in pancreatic juice has also been an area of study. Mutant P53 was found in the pancreatic juice of individuals with PanIN 2-3, intermediate and high-grade IPMN, and invasive malignancy. ${ }^{5}$ A potential novel biomarkers for early pancreatic cancer diagnosis: First, antibody microarrays using sequential plasma samples from GEMM with pancreatic cancer. Second, the presence of the heparan sulfate proteoglycan glypican 1 on the outer layer of circulating exosomes has been observed in both GEMM and patients with earlystage disease, suggesting that exosome analysis could be useful for early diagnosis. ${ }^{10}$

\section{Cancer Stem Cells}

The poor prognosis of pancreatic cancer may be 
caused by a prevalence of Cancer Stem Cells (CSCs). Cancer stem cells are a population of cancer cells showing stem cell-like characteristics. Cancer stem cells have the ability to self-renew and may initiate tumorigenesis. The expression of pancreatic cancer stem cell markers such as CD133, CD24, CD44, DCLK1, CXCR4, ESA, Oct4, and ABCB1 could be negative prognostic factors in pancreatic cancer and are responsible for its faster progression and its resistance to standard treatment. ${ }^{9}$

Expression of these factors may be responsible for reduced five-year survival and overall survival. In addition, it is evident that the expression from CD24, CD133, DCLK1, CD44, CXCR4, and Oct4 is associated with a higher incidence of lymph node metastases, whereas CD24 is also responsible for microscopic, lymphatic, venous and nerve invasion. The expression of CD44, CD24, ESA, and CD133 could also be largely involved in the resistance of $P C$ to gemcitabine therapy. ${ }^{9}$

\section{Hepatobiliary Tests and Other Blood Tests}

Other presentations of pancreatic cancer include obstructive jaundice, when a tumor in the head of the pancreas obstructs the common bile duct, and malabsorption. Biochemical tests of pancreatic function are rarely useful in diagnosis. ${ }^{18}$ Serum amylase and/or lipase levels are elevated in less than $50 \%$ of patients with resectable pancreatic cancers. A study by Asamer et al. showed that plasma amylase was an independent prognostic factor in metastatic pancreatic carcinoma. Amylase may be a novel and useful marker for patient stratification. Elevated plasma amylase is an adverse prognostic marker in patients with metastatic pancreatic cancer. ${ }^{19}$ The pancreas secretes the lipase enzyme into the duodenum to break down triglycerides into fatty acids. Amylase is an enzyme secreted by pancreatic acinar cells into the pancreatic duct and duodenum. Amylase helps the catabolism of carbohydrates into simple sugar components in the gut. Together with amylase, lipase appears in the blood vessel damage or disease associated with pancreatic acinar cells. ${ }^{20}$ Patients with obstructive jaundice show significant elevations in serum bilirubin (conjugated and total), alkaline phosphatase, gamma-glutamyl transferase, and, to a lesser extent, aspartate transaminase, and alanine transaminase. ${ }^{7,10}$ Other blood test that is very useful in managing patients with pancreatic cancer is fasting blood glucose and hemoglobin A1c levels. Paracrine effects by cancer and/or stellate cells and destruction of the pancreatic parenchyma, including the islets of Langerhans can cause diabetes mellitus. ${ }^{10}$

\section{CONCLUSION}

Pancreatic cancer usually refers to ductal adenocarcinomas of the pancreas. The different incidence of pancreatic cancer between countries also shows the important role of environmental factors for the disease. The risk factors are divided into non-modifiable and modifiable risk factors. The recent sequencing of the pancreatic cancer genome has confirmed that four genes are most commonly affected by somatic mutation in this neoplasm: KRAS, CDKN2A/p16, SMAD4, and P53). The development and progression of pancreatic cancer is a multistep process. Precursor lesions for pancreatic adenocarcinoma include Intraepithelial Pancreatic Mucinous Neoplasms (IPMNs) and Pancreatic Intraepithelial Neoplasia (PanIN). Pancreatic cancer rarely manifests specific signs or symptoms. Because of its poorness of symptoms, it is often diagnosed in an advanced stage. The low positive predictive value of CA 19-9 indicates that it cannot be used in screening. CA19-9 lacks sensitivity or specificity for early pancreatic cancer diagnosis, despite its routine use to monitor disease progression, recurrence, and/or therapy response. Both CEA and CA 19-9 are elevated in some pancreatic cancer. Biochemical tests of pancreatic function are rarely useful in diagnosis. Serum amylase and/or lipase levels are elevated in less than $50 \%$ of patients with resectable pancreatic cancers. Patients with obstructive jaundice show significant elevations in serum bilirubin (conjugated and total), alkaline phosphatase, gamma-glutamyl transferase, and, to a lesser extent, aspartate transaminase, and alanine transaminase.

\section{REFERENCES}

1. Kumar V, Abbas AK, Aster JC. Robbins basic pathology. $10^{\text {th }}$ Ed., Philadelphia, Elsevier, 2018; 686-9.

2. United States of America. Source Gkobacan 2020. International Agency for Research on Cancer. World Health Organization. The Global Cancer Observatory, 2021; 1 -3.

3. Indonesia. Source Gkobacan 2020. International Agency for Research on Cancer. World Health Organization. The Global Cancer Observatory, 2021; 1-2.

4. Asia. Source Gkobacan 2020. International Agency for Research on Cancer. World Health Organization. The Global Cancer Observatory, 2021; 1-2.

5. McGuigan, Kelly P, Turkington R, Jones C, Coleman HG, McCain RS. Pancreatic cancer: A review of clinical diagnosis, epidemiology, treatment and outcomes. World J Gastroenterol, 2018; 24(43): 4846-61.

6. Capasso M, Franceschi M, Castro KIR, Crafa P, Cambie 
G. Epidemiology and risk factors of pancreatic cancer. Acta Biomed, 2018; 89(9): 141-6.

7. South-Eastern Asia. Source Gkobacan 2020. International Agency for Research on Cancer. World Health Organization. The Global Cancer Observatory. 2021; $1-2$.

8. Lahero D, Pancreatic cancer. In: Goldman L, Schafer AI. Goldman-Cecil Medicine. Philadelphia, Elsevier, 2020; 1305-7.

9. Gzil A, Zarebska I, Burslewiicz W, Antosik P, Grzanka D, Szylberg L. Markers of pancreatic cancer stem cells and their clinical and therapeutic implication. Mol Biol Rep, 2019; 46(6): 6629-45.

10. Kleef J, Korc M, Apte $M$, Vecchia $C L$, Johnson $C D$, Biankin AV. Pancreatic cancer. Nature Review, 2016; 2:1-22.

11. Dreyer SB, Chabg DK, Bailey P, Biankin AV. Pancreatic cancer genomes: Implication for clinical management and therapeutic development. Clin Cancer Res, 2017; 23(7): 1638-46.

12. Shires GT, Wilfong LS. Pancreatic cancer, cystic pancreatic neoplasma. In: Sleisenger and Fordtran's Gastrointestinal and Liver Disease. $10^{\text {th }}$ Ed., Philadelpia, Elsevier, 2016; 1027-43.

13. Siddiqi HA, Salwen MJ, Shaikh MF, Bownw WB. Laboratory diagnosis of gastrointestinal and pancreatic disorder. In: McPherrson RA, Pincu MR.
Henry's clinical diagnosis and management by laboratory methods. St.Louis, Elsevier, 2017; 306-318.

14. Gu D, Schlotman KE, Xie J. Deciphering the role of hedgehog signaling in pancreatic cancer. The Journal of Biomedical Research, 2016, 30(5): 353-360.

15. Khan MA. Azim S, Zubair H, Bhardwaj A, Pate GK, Khusman M. Molecular drivers of pancreatic pathogenesis: Looking inward to move foreward. Int.J.Mol.Sci, 2017; 18(779): 1-25.

16. Borazanci E, Dang CV, Robey RW, Bates SE, Chabot JA, Von Hoff DV. Pancreatic cancer: A riddle wraped: In a mystery inside an enigma. Clin Cancer Res, 2017; 23(7): 1629-37.

17. Marshal WJ, Lapsley M, Day A, Shipman K. Clinical chemistry. $9^{\text {th }}$ Ed., St.Louis, Elsevier, 2021; 127-130.

18. Sturgeon A.Tumor markers. In: Tietz fundamental of clinical chemistry and molecular diagnostic. $8^{\text {th }}$ Ed., St Louis Missouri, Elsevier, 2019; 331-58.

19. Asamer E, Szkandera J, Gibiser P, Lembeck AL, Stojakovic T. Elevated amylase in plasma represents adverse prognostic marker in patients with metastatic pancreatic cancer. Wlen Klin Wochenschr, 2018; 130: 569-74.

20. Flora Melda F, Rahajo B, Arthamin MZ. Pancreatic cancer in 31 years old patient with normal serum amilase level. Indonesian Journal of Clinical Pathology and Medical Laboratory, 2016; 23(1): 96-101. 